\title{
Influenza: One Health in action
}

\author{
Dominic E. Dwyer ${ }^{\mathrm{A}, \mathrm{C}}$ and Peter D. Kirkland ${ }^{\mathrm{B}}$ \\ ${ }^{\mathrm{A}}$ Centre for Infectious Diseases and Microbiology Laboratory \\ Services, Westmead Hospital \\ ${ }^{\mathrm{B}}$ Elizabeth McArthur Agriculture Institute \\ ${ }^{\mathrm{C}}$ Corresponding author.Email: dominic.dwyer@sydney.edu.au
}

\begin{abstract}
Influenza highlights the relevance of One Health, where experts in animal, human and environmental health combine to solve interrelated problems. Human disease due to pandemic (H1N1) 2009 influenza and avian and human disease due to influenza A/H5N1 are recent examples of new zoonoses with significant global impact. Management and prevention of influenza and other emerging infectious diseases requires the expansion and continuing support of collaborations between human and animal health experts at the clinical, diagnostic laboratory, public health, research and training levels.
\end{abstract}

A strong driver of One Health was the emergence of the new influenza viruses A/H5N1 and A/H7N7 from birds, ${ }^{1}$ as well as other respiratory viruses, such as severe acute respiratory syndrome (SARS) and henipa viruses from bats. $^{2,3}$ The appearance of the pandemic (H1N1) 2009 influenza virus further galvanized discussions about the need for understanding the interactions between human and animal health. ${ }^{4}$ The general population understand these connections, as 'bird flu' and 'swine flu' have entered the lexicon in the past few years.

The clues to the origins of human pandemics are found in the study of influenza viruses in birds and animals, study that is justified by the local and global economic, social and health impacts of influenza. This paper describes the emergence of the two influenza viruses, influenza A/H5N1 and pandemic (H1N1) 2009, that have caused recent international public health concern, and considers how an understanding of One Health contributes to managing future influenza epidemics in Australia.

\section{Influenza viruses}

Influenza viruses are naturally present in migratory water birds and spillover into human (and animal) populations may cause pandemics. The influenza viruses are members of the Orthomyxoviridae family, enveloped viruses that contain a segmented single-stranded RNA genome. The important antigenic features of influenza are the external glycoproteins, haemagglutinin $(\mathrm{H})$, responsible for virus attachment to the target cell, and neuraminidase $(\mathrm{N})$, needed for virion maturation and release. The sixteen $\mathrm{H}$ and nine $\mathrm{N}$ influenza $\mathrm{A}$ subtypes all circulate, often asymptomatically, in water birds. ${ }^{5}$ Some viruses move across into domestic poultry, with $\mathrm{A} / \mathrm{H} 5 \mathrm{~N} 1$ and $\mathrm{A} / \mathrm{H} 7 \mathrm{~N} 7$ strains particularly associated with high pathogenicity. Influenza A subtypes can infect and become established in animals, including horses (as demonstrated by the costly equine influenza $\mathrm{A} / \mathrm{H} 3 \mathrm{~N} 8$ outbreak in eastern Australia in 2007) and pigs. ${ }^{6}$ In 2010, an A/H10N7 virus affected poultry in New South Wales (NSW), with zoonotic transfer to a number of humans in close contact with infected poultry. However, only three $\mathrm{H}(\mathrm{H} 1, \mathrm{H} 3, \mathrm{H} 2)$ and two N (N1 and N2) influenza A subtypes independently circulate in humans. Influenza B viruses are only present in humans.

The two influenza viruses of most public health concern in recent years have been influenza $\mathrm{A} / \mathrm{H} 5 \mathrm{~N} 1$ and pandemic (H1N1) 2009.

\section{Influenza A/H5N1}

Influenza A/H5N1, first isolated in 1996 from a goose in Guangdong Province in China, caused severe poultry losses and occasional human infections in Hong Kong in 1997. The main human public health response that controlled this outbreak was an aggressive poultry cull. However, from 2003 the virus has moved throughout south east and eastern Asia, to Russia, central Asia and the Middle East (in 2005), Europe (2005), Africa (2006) and the Indian subcontinent (2006), making it the largest recorded epizoonosis in poultry (both commercial and backyard) and migratory birds. The combination of legal and illegal poultry and wild bird trade, and migratory water birds, contributed to its rapid spread. ${ }^{7}$ This has caused significant economic and social impact in affected countries.

Influenza $\mathrm{A} / \mathrm{H} 5 \mathrm{~N} 1$ has infected humans following contact with infected poultry, causing severe disease with a high mortality. Fortunately, human-to-human transmission is rare, preventing pandemic spread of this potentially devastating pathogen. ${ }^{8}$

Concerns about the spread of avian influenza A/H5N1 and the 2003 SARS outbreak dominated pandemic planning until the emergence in 2009 of 'swine flu'. These concerns are reflected in the Australian Health Management Plan for 
Pandemic Influenza, ${ }^{9}$ and the World Health Organization (WHO) pandemic plan. ${ }^{10}$

\section{The pandemic (H1N1) 2009 influenza virus}

The first descriptions of pandemic (H1N1) 2009 influenza virus infection occurred in the southwestern United States and Mexico in April 2009. ${ }^{11}$ This virus, quickly given the moniker 'swine flu', was identified to have animal origins, with reassortment of influenza gene segments from North American and Eurasian swine, avian and human viruses. Although seasonal $\mathrm{A} / \mathrm{H} 1 \mathrm{~N} 1$ viruses had been circulating for many years, this novel reassortant $\mathrm{A} / \mathrm{H} 1 \mathrm{~N} 1$ virus was not covered by current seasonal influenza vaccines. ${ }^{12}$ As the world population was not immune to the pandemic (H1N1) 2009 influenza virus, it spread quickly. The first Australian human case was identified in Queensland on 8 May 2009 in a traveler returning from North America, but the first evidence of significant community transmission was identified in Victoria in late May and early June 2009. ${ }^{13,14}$ The epidemic then spread nationally, taking approximately 7 weeks for peak activity to be reached in Western Australia. There was variability in urban and rural attack rates, and within cities. For example, in Sydney, activity was focused in the western and southwestern suburbs, with rates of hospitalisation due to pandemic (H1N1) 2009 influenza virus infection approximately three times higher than those seen in the eastern and northern regions of the city. ${ }^{15}$

The origins and exact timing of the emergence of this new pandemic virus remain uncertain. It may have been circulating, but unrecognised, in pigs for some years, as there is minimal influenza surveillance in most commercial pig populations. Interestingly, transmission of the pandemic (H1N1) 2009 influenza virus from humans to pigs has occurred in Australia and other countries. This is, however, not unique. In the United States a number of strains of influenza $\mathrm{A}$ are endemic in the pig population and have been frequently transferred to humans and vice versa.

For the most part, pandemic (H1N1) 2009 influenza virus infection caused a relatively mild disease. However hospital or intensive care unit (ICU) admissions, and deaths, were proportionally higher in younger populations compared to seasonal influenza. Risk factors for severe pandemic (H1N1) 2009 influenza infection included morbid obesity, pregnancy and immunosuppression, although approximately one-third of ICU admissions and deaths occurred in otherwise healthy individuals. ${ }^{16,17}$

A locally produced monovalent pandemic (H1N1) 2009 influenza vaccine was available after the first pandemic wave in Australia, and now a trivalent formulation that includes pandemic (H1N1) 2009, seasonal A/H3N2 and $B$ viruses has been distributed worldwide. Although vaccination accessibility and uptake varies significantly between countries, the 'herd' immunity induced by infection during the first and subsequent waves, and the availability of specific vaccination (and possibly use of antiviral drugs) mean that the subsequent waves are likely to be less severe than those seen during earlier pandemics.

\section{Where to from here?}

What do the concepts of One Health mean for managing future influenza epidemics in Australia, using pandemic (H1N1) 2009 influenza and influenza A/H5N1 as examples? How should these concepts be supported? Some of the approaches to enhancing One Health are listed in Box 1.

\section{Box 1. One Health approaches to influenza}

- Enhanced surveillance

- clinical human and veterinary disease

- combination of clinical and laboratory surveillance

- co-ordination of local, regional, national and international surveillance programs

- genetic and antigenic variation of viruses.

- Rapid, 'real time' dissemination of surveillance data.

- Understanding patterns of transmission: human-tohuman (and animal), animal-to-animal (and human).

- Understanding pathogenesis of disease in animals and humans.

\section{Clinical surveillance}

In human health, clinical networks in Australia allowed the risk factors and clinical presentations of severe pandemic (H1N1) 2009 influenza to be identified and managed quickly. ${ }^{16-18}$ Advances in diagnostic laboratory techniques meant that diagnoses and characterisation of the pandemic (H1N1) 2009 influenza virus, including antiviral drug resistance, was rapid. ${ }^{19-21}$

With most respiratory tract viral infections there is more mild (or asymptomatic, or non-specific) disease than severe clinical disease. This means that serological testing is needed to determine the true rate of influenza infection. ${ }^{22}$ The impact of infection varies by season, age group and underlying health status. This makes all-encompassing clinical surveillance difficult, especially as laboratory testing is needed to differentiate the various viral causes of disease. The quality of human surveillance for clinical presentations of new respiratory tract infections in Australia is patchy. Investment in comprehensive surveillance systems that capture, in real time, severe disease (e.g. ICU admissions, or hospitalisations for pneumonia) or outbreaks (e.g. in aged-care facilities) is required. 


\section{Laboratory surveillance}

As influenza is a notifiable disease (and pandemic (H1N1) 2009 and A/H5N1 are quarantinable diseases) in NSW, passive (but not active) laboratory surveillance is already in place. The widespread use of rapid antigen tests and nucleic acid testing has improved clinical diagnosis of influenza. Paradoxically this may cause a problem: virus isolation is now performed less frequently, but isolates are needed for antigenic and genetic surveillance of influenza strains. $^{23,24}$

Worldwide, surveillance of influenza isolates is undertaken by the WHO Global Influenza Network, which consists of five Collaborating Centres and some 110 National Influenza Centres. Australia has a WHO Collaborating Centre in Melbourne and three National Influenza Centres: in Sydney (Institute of Clinical Pathology and Medical Research, Westmead), Melbourne (Victorian Infectious Diseases Reference Laboratory) and Perth (PathWest Laboratory Medicine WA). These, and other laboratories in the Australian Public Health Laboratory Network and in the Asia-Pacific region, contribute influenza strains to the WHO Collaborating Centre in Melbourne and contribute to the international surveillance of influenza strains.

\section{Veterinary surveillance}

In contrast to the situation in birds and pigs in most overseas countries, the Australian animal and bird populations are currently believed to be free of infection with highly pathogenic influenza viruses. ${ }^{25}$ The exception is likely to be semi-free range duck populations that have regular opportunity for contact with wild aquatic birds. Generally, Australian chicken populations are completely free of infection with influenza A viruses. When A/H5 or A/H7 infections have occurred in poultry, overt disease outbreaks have rapidly followed. These have been quickly followed by interventions, usually with bird depopulation of the infected farms. The current knowledge of risks posed to humans from infected birds and the established links between animal and public health agencies reduce the chance of human disease.

Even though Australian pigs have been free of infection and so are highly susceptible, pandemic (H1N1) 2009 influenza virus infection mostly passed unnoticed, with little or no disease. In most situations, people posed a greater risk to infection in pigs than the converse. The $\mathrm{A} / \mathrm{H} 3 \mathrm{~N} 8$ virus that infected horses and dogs in Australia presented many challenges for the veterinary profession and laboratories but passed unnoticed from a public health perspective. Despite exposures to massive doses of virus, there was no record of human infection.

From a laboratory perspective, the A/H5N1 epidemics that occurred overseas were invaluable as a stimulus for enhancing the diagnostic capacity and preparedness of veterinary and public health laboratories. In addition, communication and interaction between human and animal health laboratories has improved. Ongoing support of human and veterinary laboratories is required, especially to undertake the specialised reference laboratory analyses needed to characterise new outbreaks.

\section{Human and veterinary collaborations}

Continually enhancing the communication between the various national and state human and animal health groups responsible for influenza and communicable diseases will assist in supporting rapid containment of zoonotic threats, and to this end there is veterinary representation on committees such as the Australian Public Health Laboratory Network and the Communicable Diseases Network of Australia. Expansion of collaborations between human and animal health experts should be encouraged at the clinical, diagnostic laboratory, research and training levels. An example of this in NSW is the formation in 2010 of the Sydney Emerging Infections and Biosecurity Institute (located at the Sydney Medical School and Westmead Hospital), a multi-disciplinary and multi-faculty approach to One Health.

\section{Conclusion}

While the One Health stimulus from recent epidemic/ pandemic events may be abating recent experience has demonstrated that well-coordinated animal and human surveillance for influenza viruses (and other respiratory pathogens) is required for pandemic planning and management.

\section{References}

1. Monto AS. The threat of an avian influenza pandemic. $N$ Engl $J$ Med 2005; 352(4): 323-5. doi:10.1056/NEJMp048343

2. Ksiazek TG, Erdman D, Goldsmith CS, Zaki SR, Peret T, Emery $\mathrm{S}$ et al. A novel coronavirus associated with severe acute respiratory syndrome. $N$ Engl J Med 2003; 348(20): 1953-66. doi:10.1056/NEJMoa030781

3. Field HE, Mackenzie JS, Daszak P. Henipaviruses: emerging paramyxoviruses associated with fruit bats. Curr Top Microbiol Immunol 2007; 315: 133-59. doi:10.1007/978-3-540-70962-6_7

4. Powdrill TF, Nipp TL, Rinderknecht JL. One health approach to influenza: assessment of critical issues and options. Emerg Infect Dis 2010; 16(8): e1. doi:10.3201/eid1608.100673

5. Hampson AW. Influenza virus antigens and 'antigenic drift'. In: Potter CW, editor. Influenza. Perspectives in Medical Virology. 7th ed. Amsterdam: Elsevier; 2002. pp. 49-85.

6. Webster RG, Bean WJ, Gorman OT, Chambers TM, Kawaoka Y. Evolution and ecology of influenza A viruses. Microbiol Rev 1992; 56(1): 152-79.

7. Peiris JS, de Jong MD, Guan Y. Avian influenza virus (H5N1): a threat to human health. Clin Microbiol Rev 2007; 20(2): 243-67. doi:10.1128/CMR.00037-06

8. Beigel JH, Farrar J, Han AM, Hayden FG, Hyer R, de Jong MD et al. Avian influenza A (H5N1) infection in humans. N Engl $J$ Med 2005; 353(13): 1374-85. doi:10.1056/NEJMra052211 
9. Australian Government Department of Health and Ageing. Australian Health Management Plan for Pandemic Influenza. 2008. Available from: http:/www.flupandemic.gov.au/internet/ panflu/publishing.nsf/Content/ahmppi-1 (Cited 12 Feburary 2010).

10. World Health Organization. Pandemic influenza preparedness and response: a WHO guidance document. April 2009. Available from: http://www.who.int/csr/disease/influenza/ pipguidance2009/en/ (Cited 7 May 2011.)

11. Centers for Disease Control and Prevention (CDC). Swine influenza A (H1N1) infection in two children - southern California, March-April 2009. MMWR Morb Mortal Wkly Rep 2009; 58(15): 400-2.

12. Novel Swine-Origin Influenza A. (H1N1) Virus Investigation Team. Emergence of a novel swine-origin influenza A (H1N1) virus in humans. N Engl J Med 2009; 360(25): 2605-15. doi:10.1056/NEJMoa0903810

13. Appuhamy RD, Beard FH, Phung HN, Selvey CE, Birrell FA, Culleton TH. The changing phases of pandemic (H1N1) 2009 in Queensland: an overview of public health actions and epidemiology. Med J Aust 2010; 192(2): 94-7.

14. Australian Government Department of Health and Ageing. Australian influenza report no. 21 - 26 September to 2 October 2009. Available from: http://www.health.gov.au/internet/ main/publishing.nsf/Content/cda-ozflu-no21-09.htm (Cited 20 November 2009.)

15. Dwyer DE. Lessons from the southern hemisphere: the first wave of the 2009 influenza pandemic in Australia. In: Scheld WM, Grayson ML, Hughes JM, editors. Emerging Infections. 9th ed. Washington DC: American Society of Microbiology Press; 2010. pp. 1-16.

16. ANZIC Influenza Investigators. Webb SA, Pettilä V, Seppelt I, Bellomo R, Bailey M. Critical care services and 2009 H1N1 influenza in Australia and New Zealand. N Engl J Med 2009; 361(20): 1925-34. doi:10.1056/NEJMoa0908481

17. Tramontana AR, George B, Hurt AC, Doyle JS, Langan K, Reid $\mathrm{AB}$ et al. Oseltamivir resistance in adult oncology and hematology patients infected with pandemic (H1N1)
2009 virus, Australia. Emerg Infect Dis 2010; 16(7): 1068-75. doi:10.3201/eid1607.091691

18. Australia and New Zealand Extracorporeal Membrane Oxygenation (ANZ ECMO) Influenza Investigators. Extracorporeal Membrane Oxygenation for 2009 influenza A (H1N1) acute respiratory distress syndrome. JAMA 2009; 302(17): 1888-95. doi:10.1001/jama.2009.1535

19. Blyth CC, Iredell JR, Dwyer DE. Rapid-test sensitivity for novel swine-origin influenza A (H1N1) virus in humans. N Engl J Med 2009; 361(25): 2493. doi:10.1056/NEJMc0909049

20. Kok J, Blyth CC, Foo H, Patterson J, Taylor J, McPhie K et al. Comparison of a rapid antigen test with nucleic acid testing during cocirculation of pandemic influenza A/H1N1 2009 and seasonal influenza A/H3N2. J Clin Microbiol 2010; 48(1): 290-1. doi:10.1128/JCM.01465-09

21. Wang B, Dwyer DE, Blyth CC, Soedjono M, Shi H, Kesson A et al. Detection of the rapid emergence of the H275Y mutation associated with oseltamivir resistance in severe pandemic influenza virus A/H1N1 09 infections. Antiviral Res 2010; 87(1): 16-21. doi:10.1016/j.antiviral.2010.04.002

22. Gilbert GL, Cretikos MA, Hueston L, Doukas G, O'Toole B, Dwyer DE et al. (H1N1) 2009 antibodies in residents of New South Wales, Australia, after the first pandemic wave in the southern hemisphere winter. PLoS ONE 2010; 5(9): e12562. doi:10.1371/journal.pone.0012562

23. Blyth CC, Kelso A, McPhie KA, Ratnamohan VM, Catton M, Druce JD et al. The impact of the pandemic (H1N1) 2009 virus on seasonal influenza A viruses in the southern hemisphere, 2009. Euro Surveill 2010; 15(31): pii: 19631.

24. Hurt AC, Ernest J, Deng YM, Iannello P, Besselaar TG, Birch C et al. Emergence and spread of oseltamivir-resistant A(H1N1) influenza viruses in Oceania, South East Asia and South Africa. Antiviral Res 2009; 83(1): 90-3. doi:10.1016/j.antiviral.2009. 03.003

25. Hansbro PM, Warner S, Tracey JP, Arzey KE, Selleck P, O'Riley K et al. Surveillance and analysis of avian influenza viruses, Australia. Emerg Infect Dis 2010; 16(12): 1896-904. 